\title{
ОЦЕНКА ЭНЕРГОВЫДЕЛЕНИЯ В МИШЕНИ С УРАНСОДЕРЖАШИМ МАТЕРИАЛОМ ПРИ ПРОИЗВОДСТВЕ 99 МО С ИСПОЛЬЗОВАНИЕМ МИШЕНЕЙ УСОВЕРШЕНСТВОВАННОЙ КОНСТРУКЦИИ НА РЕАКТОРЕ ВВР-Ц
}

\author{
0.Ю. Кочнов *, В.В. Колесов * , Р.В. Фомин * * \\ * Обнинский филиал ГНЦ РФ НИФХИ Им. Л.Я. Карпова, г. Обнинск \\ * * Обнинский институт атомной энергетики нияу МИФИ, г. Обнинск
}

Увеличение производства ${ }^{99}$ Мо в условиях мирового дефицита является актуальной задачей. При переходе на новую мишень проточного типа [1] для наработки ${ }^{99}$ Мо возникла необходимость оценки энерговыделения для обоих типов мишеней. Результаты расчетов показали значительное увеличения энерговыделения для модернизированной мишени.

Ключевые слова: реактор ВВР-ц, ${ }^{99}$ Мо, энерговыделение.

Key words: WWR-c reactor, ${ }^{99} \mathrm{Mo}$, energy deposition.

\section{ВВЕДЕНИЕ}

В качестве расчетной модели для определения энерговыделения в мишенях использовалась прецизионная трехмерная модель активной зоны реактора ВBР4 [2].

Мишень для производства осколочного ${ }^{99}$ Мо должна удовлетворять следующим требованиям:

- иметь размеры и конструкцию, которые позволяют обеспечить ее загрузку в канал ядерного реактора и выгрузку из него;

- содержать достаточное количество ${ }^{235} \mathrm{U}$;

- обеспечивать барьер, препятствующий выбросу радиоактивных продуктов и особенно газов во время и после облучения;

- конструкция мишени должна быть легко разборной в условиях горячей камеры;

- иметь конструкцию и состав, обеспечивающие ее химическую переработку в горячей камере за минимальное время.

Целесообразно, чтобы в процессе радиохимической переработки мишени количество жидких радиоактивных отходов было минимальным. Длительность переработки урановой мишени существенным образом влияет на выход ${ }^{99}$ Мо. Из-за распада каждый час теряется около $1,5 \%$ образовавшегося в мишени ${ }^{99}$ Мо. Поэтому операции с мишенью при ее разделке в горячей камере должны быть достаточно простыми и легко выполнимыми.

(c) 0.Ю. Кочнов, В.В. Колесов, Р.В. Фомин, 2012 
В настоящее время для производства осколочного ${ }^{99}$ Мо используется мишень, содержащая уран высокого обогащения (около $90 \%$ ) по ${ }^{235} \mathrm{U}$.

\section{ПОСТАНОВКА ЗАДАЧИ}

На рисунках 1-3 приводятся конструкции и расположения стандартной используемой в настоящее время мишени типа «стакан в стакане». Конструкция мишени, помимо удовлетворения основным требованиям при работе в реакторе, ориентирована на удобство переработки ее в условиях горячей камеры.

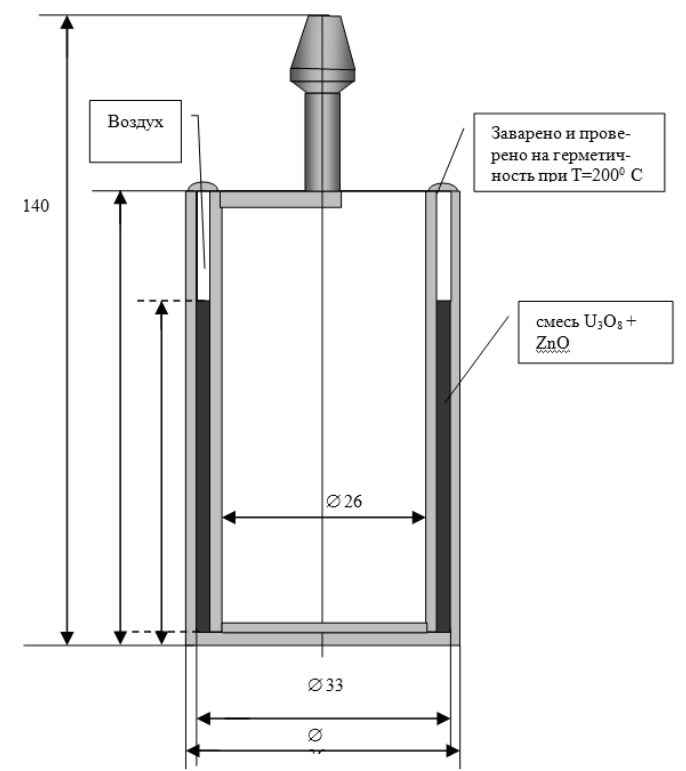

Рис. 1. Конструкция стандартной мишени для производства ${ }^{99}$ Мо типа «стакан в стакане»

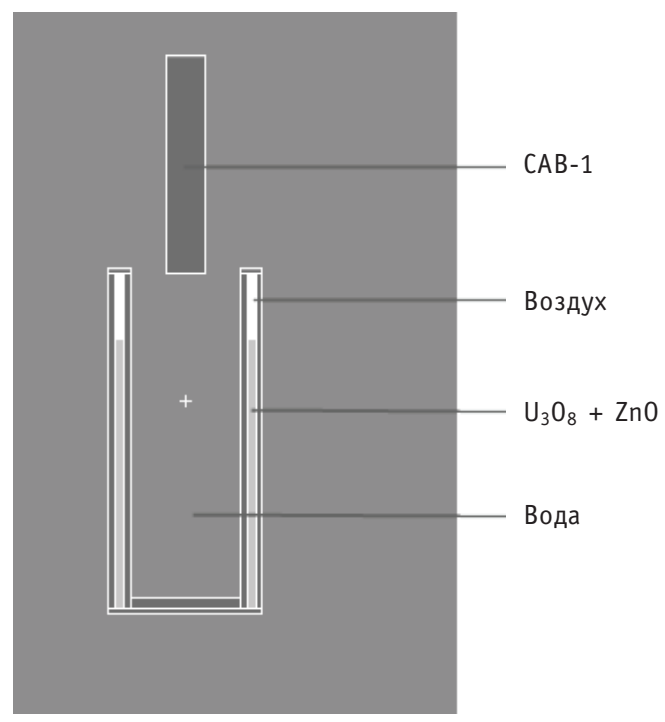

Рис. 2. Детализация стандартной мишени для производства ${ }^{99}$ Мо в модели активной зоны реактора ВBР-ц 


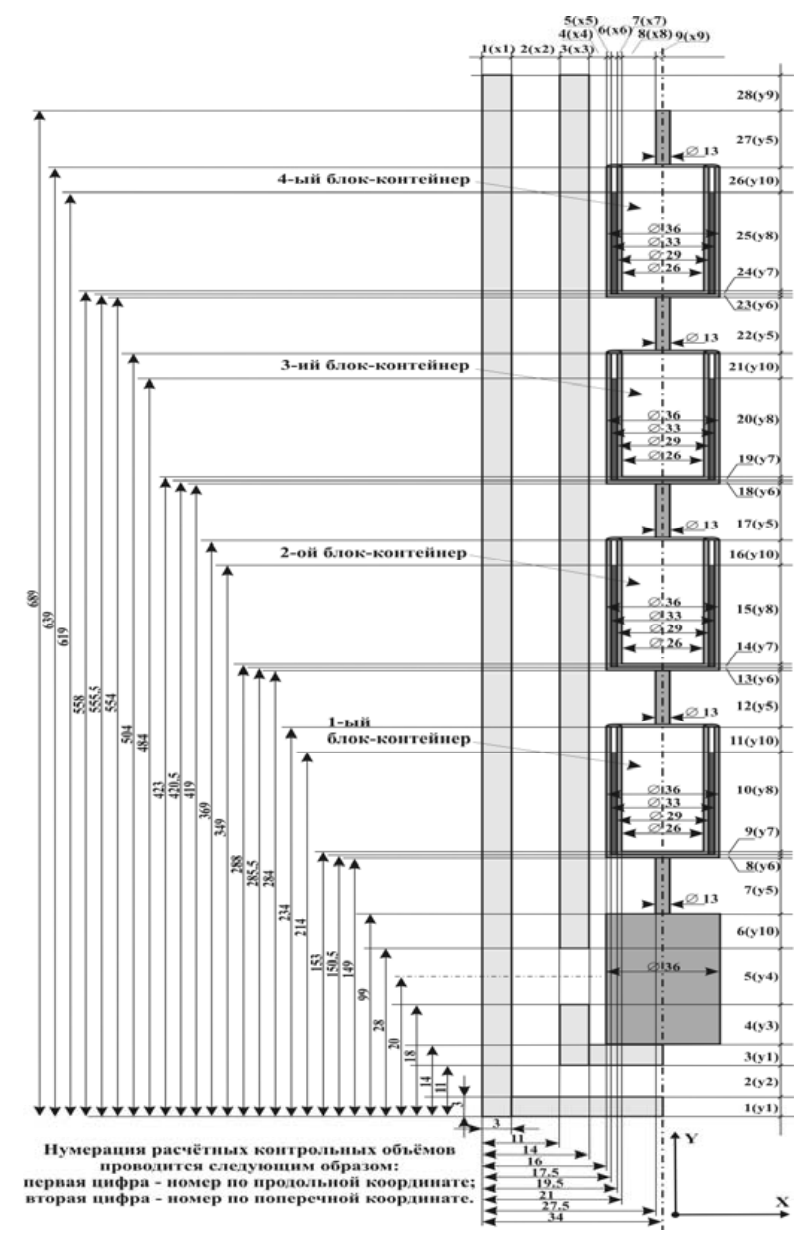

Рис. 3. Расположение стандартных мишеней в экспериментальном канале

Загрузка смеси в мишень осуществляется с помощью разработанного механического устройства. Одновременно с загрузкой происходит уплотнение засыпки. После заварки мишени осуществляется проверка ее на герметичность при температуре $200^{\circ} \mathrm{C}$. Конструкция мишени оказалась работоспособной и не претерпела существенных изменений за весь период наработки ${ }^{99}$ Мо в институте. Изменялись лишь величина загрузки урана и состав компонента разбавителя.

Мишень может быть установлена как в экспериментальный канал с естественной циркуляцией, так и в каналы с принудительным охлаждением. При этом загрузка мишени по ${ }^{235} \mathrm{U}$ может отличаться более чем в 10 раз. Для увеличения наработки ${ }^{99}$ Мо в канале дальнейшая модернизация существующей конструкции мишени была направлена на увеличение в ней количества загруженного урана. При этом были увеличены габаритные размеры мишени и предложено техническое решение, облегчающее расцепление двух цилиндров после взрезки мишени.

На рисунках 4, 5 представлена конструкция модифицированной мишени. Мишень с проточным охлаждением представляет собой трубчатую конструкцию со сквозным проходом воды для улучшения теплосъема. Между внутренней и наружной трубками помещается смесь ${ }^{235} \mathrm{U}(90 \%)$ и оксида цинка. Одновременно в канал устанавливается не более двух мишеней. Предварительные оценки показали возможность увеличения загрузки урана за счет улучшения теплоотвода от мише- 


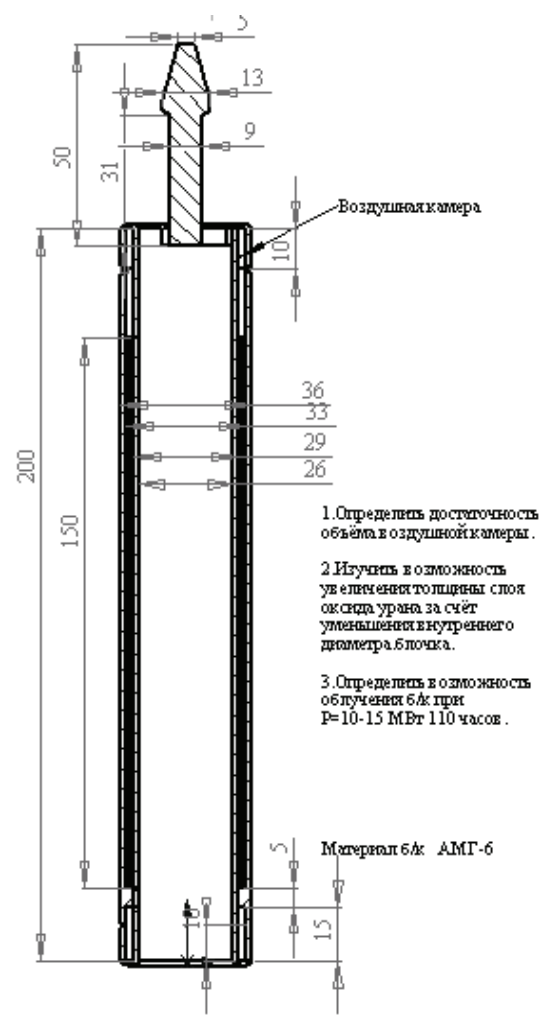

Рис. 4. Конструкция модифицированной мишени для производства ${ }^{99}$ Мо

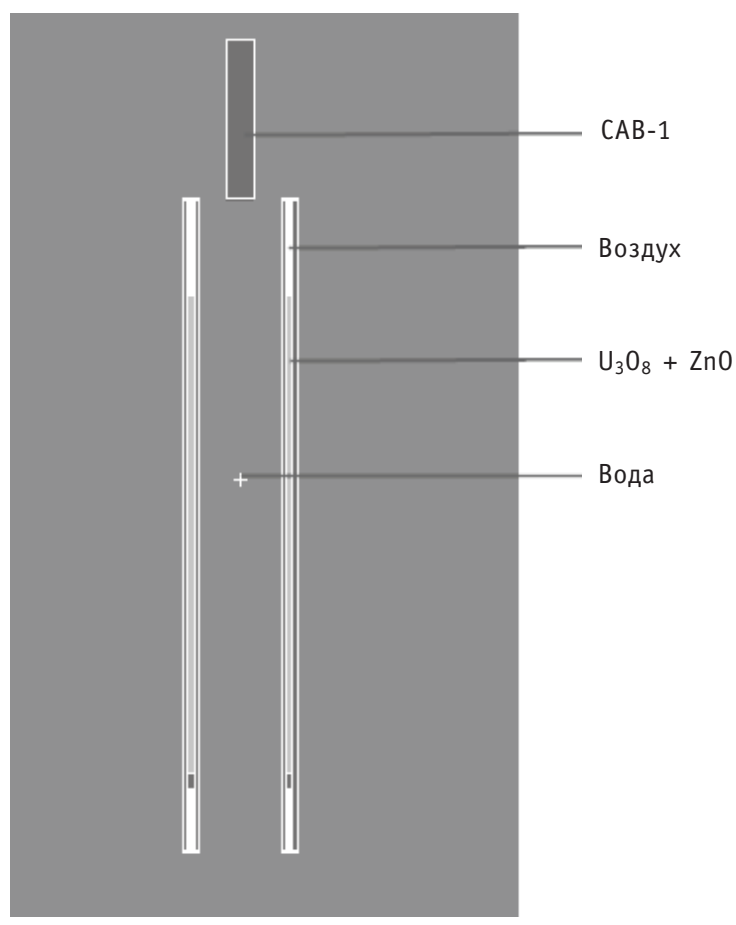

Рис. 5. Детализация модифицированной мишени для производства ${ }^{99}$ Мо в модели активной зоны реактора ВВР-ц

ни и, как следствие, увеличения наработки ${ }^{99}$ Мо в 1.7 раза по сравнению с мишенью типа «стакан в стакане».

На рисунках 6, 7 приводится горизонтальный и вертикальный разрезы части модели активной зоны с экспериментальным каналом 4-1 и установленной в нем мишенью.

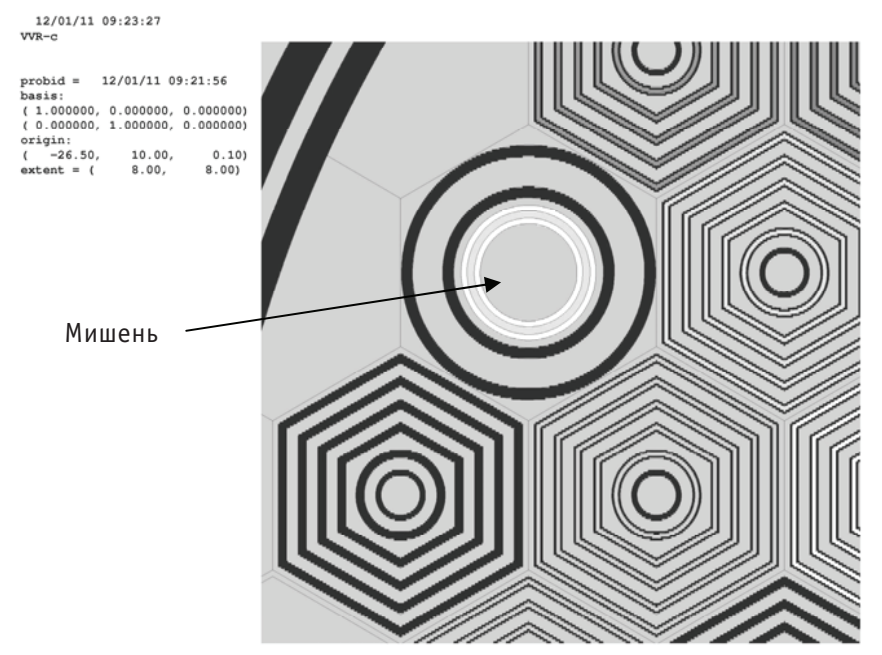

Рис. 6. Экспериментальный канал 4-1 с установленными модифицированными мишенями (горизонтальный разрез) 


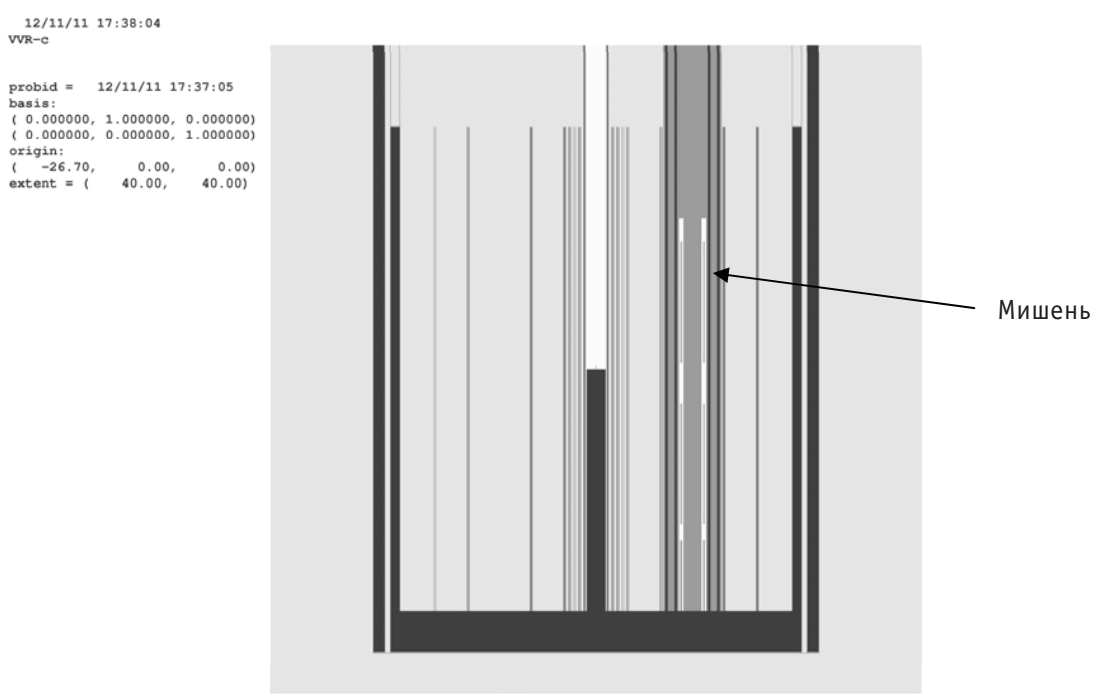

Рис. 7. Экспериментальный канал 4-1 с установленными модифицированными мишенями (вертикальный разрез)

Топливо мишени представляет собой смесь $\mathrm{U}_{3} \mathrm{O}_{8}+\mathrm{ZnO}$ с обогащением по ${ }^{235} \mathrm{U}$, равным $90 \%$, с массой $\mathrm{U}_{3} \mathrm{O}_{8}-15$ г и $\mathrm{ZnO}-75$ г. Температура топлива в мишени полагалась равной $175^{\circ} \mathrm{C}$, в активной зоне $-75^{\circ} \mathrm{C}$, тепловая мощность реактора равной 10 МВт.

\section{РЕЗУЛЬТАТЫ РАСЧЕТОВ}

На рисунках 8, 9 приводится энергетическое распределение плотности потока нейтронов в стандартной и модифицированной мишенях. Из рисунков видно, что в трех из четырех стаканов модифицированной мишени плотность потока тепловых нейтронов превышает соответствующую плотность потока тепловых нейтронов стандартной мишени. Это приводит к значительному увеличению энерговыделения в модифицированной мишени. Результаты расчетов энерговыделения в контейнерах для стандартной и модифицированной мишеней приведены в табл. 1, 2.

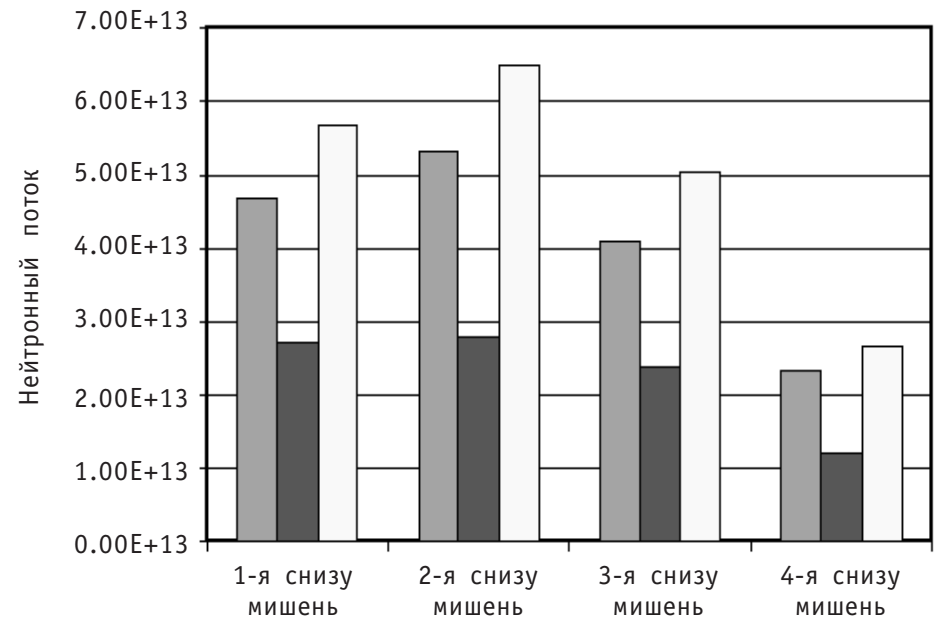

Рис. 8. Энергетическое распределение плотности потока нейтронов в каждом из четырех стаканов стандартной мишени: $\square-0-0,2$ кэВ; $\square-0,2-5$ кэВ; $\square-5$ кэВ-10 МэВ 


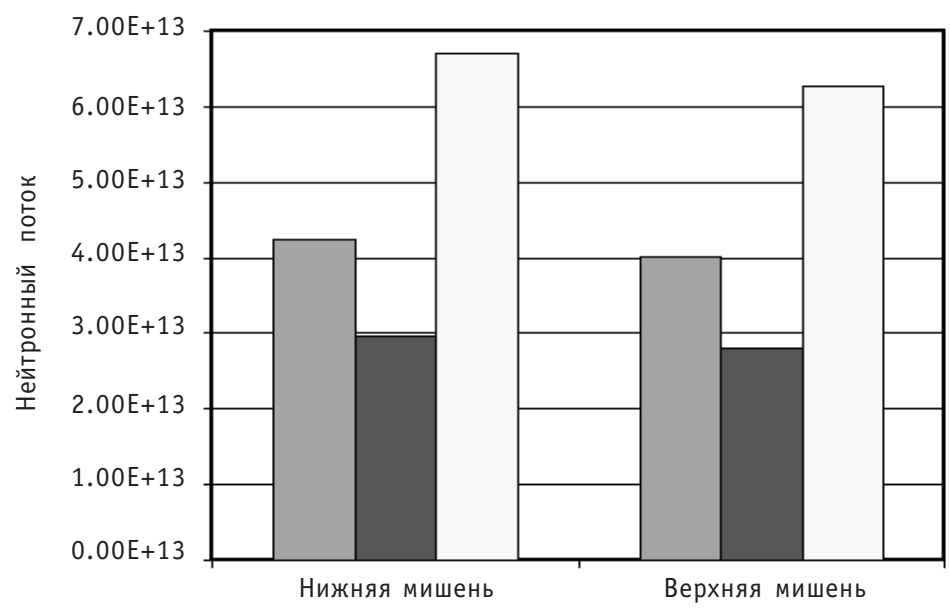

Рис. 9. Энергетическое распределение плотности потока нейтронов в каждом из двух стаканов модифицированной мишени: $\square-0-0,2$ кэВ; $\square-0,2-5$ кэВ; $\square-5$ кэВ-10 МэВ

Таблица 1

Энерговыделение в контейнерах стандартной мишени

\begin{tabular}{|c|c|c|c|}
\hline \multirow{2}{*}{$\begin{array}{c}\text { Энерговыделение от нейтронов и } \\
\text { фотонов }\end{array}$} & \multicolumn{3}{|c|}{ Модель } \\
\hline & МBт & MBT/г U-235 & $\mathrm{MBT} /\left\ulcorner\mathrm{U}_{3} \mathrm{O}_{8}\right.$ \\
\hline Первая снизу из четырех & 0.0057 & 0.00166 & 0.00126 \\
\hline Вторая снизу из четырех & 0.0062 & 0.00181 & 0.00138 \\
\hline Третья снизу из четырех & 0.0051 & 0.00149 & 0.00113 \\
\hline Четвертая снизу из четырех & 0.0026 & 0.00076 & 0.00058 \\
\hline 0бщее & 0.0196 & 0.00143 & 0.00109 \\
\hline \multicolumn{4}{|c|}{ Macca U-235 в одной мишени $=3.4242$ г, $\bigcup_{3} 0_{8}-4.5$ г. } \\
\hline$K_{\text {eff }}$ & \multicolumn{3}{|c|}{$1.00897 \pm 0.00034$} \\
\hline
\end{tabular}

\section{Энерговыделение в контейнерах}

Таблица 2 модифицированной мишени

\begin{tabular}{|c|c|c|c|}
\hline $\begin{array}{c}\text { Энерговыделение от нейтронов и } \\
\text { фотонов }\end{array}$ & МВт & МВт/г U-235 & МВт/г $\mathrm{U}_{3} \mathrm{O}_{8}$ \\
\hline Нижняя мишень & 0.0169 & 0.00148 & 0.00112 \\
\hline Верхняя мишень & 0.0160 & 0.00140 & 0.00106 \\
\hline Общее & 0.0329 & 0.00144 & 0.00109 \\
\hline Масса U-235 в одной мишени 11.4243 г, $\mathrm{U}_{3} 0_{8}-15$ г. \\
\hline$K_{\text {eff }}$ & \multicolumn{3}{|l}{$1.01097 \pm 0.00034$} \\
\hline
\end{tabular}

\section{ЗАКЛЮЧЕНИЕ}

В результате проведенных исследований показано значительное увеличение энерговыделения в мишенях новой улучшенной конструкции проточного типа.

Мощность модифицированного канала с принудительным охлаждением составила 32.9 кВт, что почти в 1.7 раза больше (19.6 кВт) энерговыделения в канале при загрузке мишеней старой конструкции типа «стакан в стакане» (см. табл. 1, 
2). Это позволяет нарабатывать большее количество радионуклида ${ }^{99}$ Мо при таких же уровне мощности и кампании реактора ВВР-ц. При этом себестоимость молибден-технециевых генераторов может быть уменьшена и, как следствие, диагностические процедуры для онкологических больных станут доступнее.

\section{Литература}

1. Kochnov O.Yu., Pozdeev V.V. Prospects for the Development of Mo-99 Production Consistent with WWR-c Reactor Modernization at the Branch of FSUE «Karpov Institute of Physical Chemistry»/ International Conf. on Research Reactors,IAEA,Morocco-2011. - P. 60-61.

2. Колесов В.В., Кочнов О.Ю., Волков Ю.В., Украинцев В.Ф., Фомин Р.В. Создание прецизионной модели реактора ВВР-цдля последующей оптимизации его конструкции инаработки ${ }^{99}$ Мо идругих радионуклидов//Известия вузов. Ядерная энергетика. - 2011. - №4. -С. 129-133.

Поступила в редакцию 28.05.2012 


\section{ABSTRACTS OF THE PAPERS}

\section{УдК 621.039.51}

New Technique to Reformat Multigroup Cross-Sections for Monte-Carlo Calculation |I.R. Suslov, I.V. Tormyshev, K.G. Mel'nikov; Editorial board of journal «Izvestia visshikh uchebnikh zavedeniy. Yadernaya energetica» (Communications of Higher Schools. Nuclear Power Engineering). - Obninsk, 2012. - 8 pages, 3 tables, 2 illustrations. - References, 21 titles.

New method to calculate equiprobability bins from Legendre expansion scattering cross-sections is proposed. The method uses a correction of equiprobability bins boundaries to provide a conservation of the first angular moment. The code CRSRD-ST to reformat cross sections from DTF to ACE format with proposed technique is developed. Numerical results shows the method proposed significantly improves an agreement between deterministic and Monte-Carlo calculations.

\section{удК 621.039.51}

Boundary Resonance Effects in the Fast Reactor with the Heterogeneous Core $\mid$ A.A. Bezborodov, E.V. Dolgov, D.A. Klinov, V.V. Kolesov, V.Yu. Stogov, I.R. Suslov, V.I. Folomeev; Editorial board of journal «Izvestia visshikh uchebnikh zavedeniy. Yadernaya energetica» (Communications of Higher Schools. Nuclear Power Engineering). - Obninsk, 2012. - 10 pages, 2 tables, 4 illustrations. - References, 10 titles.

Boundary resonance effects is considering with the application of the high-speed subgroup approximation technique employment in practical tasks for description of neutron cross-sections interactions with media nuclides nuclei in resonance part of energy for physical simulation of the fast reactor plants with the heterogeneous core.

\section{УдК 621.039.51}

Energy Deposition Evaluation in the Target with Uranium-Containing Material for the ${ }^{99} M o$ Production in WWRC Reactor with the Impruved Design of Target|O.Yu. Kochnov, V.V. Kolesov, R.V. Fomin; Editorial board of journal «Izvestia visshikh uchebnikh zavedeniy. Yadernaya energetica» (Communications of Higher Schools. Nuclear Power Engineering). - Obninsk, 2012. - 7 pages, 2 tables, 9 illustrations. - References, 2 titles.

Increased production of the ${ }^{99} \mathrm{Mo}$ in the context of rising global demand is an urgent task. The new type of target for ${ }^{99}$ Mo producing in WWR-c reactor technological channels was developed. A series of calculations to estimate energy deposition for standard and modified target were done. A considerable energy deposition increasing in modified target was found.

\section{удк 621.039 .56}

Method of ${ }^{16} \mathrm{~N}$ Generation for Test of Radiation Controlled Cannels on Nuclear Power Stations with Water-Cooled Reactors|V.A. Khryachkov, I.P. Bondarenko, P.A. Dvornikov, B.V. Zhuravlev, S.N. Kovtun, T.A. Khromyleva, A.V. Pavlov, N.G. Roschin; Editorial board of journal «Izvestia visshikh uchebnikh zavedeniy. Yadernaya energetica» (Communications of Higher Schools. Nuclear Power Engineering). - Obninsk, 2012. - 5 pages, 3 illustrations. - References, 3 titles.

The preferences of ${ }^{19} \mathrm{~F}(\mathrm{n}, \alpha)^{16} \mathrm{~N}$ nuclear reaction use for radiation control channels test on watercooled power reactors are analyzed. The new measurements for more accurate determination of ${ }^{19} \mathrm{~F}(\mathrm{n}, \alpha){ }^{16} \mathrm{~N}$ reaction cross section energy dependence have been carried out. A set of new methods for background reducing and improvement of events determination reliability was developed.

\section{удК 621.039.524.44:697.93}

Calculation-based Justification for the Algorithm of Pipelines Leak Control by Air Humidity|P.A. Dvornikov, S.N. Kovtun, A.A. Budarin, V.P. Polionov, N.N. Titarenko, D.M. Shvetsov, N.G. Roshchin, A.L. Matveev, E.L. Matveev; Editorial board of journal «Izvestia visshikh uchebnikh zavedeniy. Yadernaya energetica» (Communications of Higher Schools. Nuclear Power Engineering). - Obninsk, 2012. - 10 pages, 9 tables, 3 illustrations. - References, 9 titles.

Dynamic processes in thermal insulation of power plants pipelines caused by depressurization are the goal of the research. Simulation of various leaks was realized by using adapted computer code KUPOL-M developed in SSC RF IPPE.

\section{УДК 621.039 .58}

Analysis of the Errors Committed by NPP MCR Operators during Implementation of Operating Procedures| N.V. Pleshakova, A.N. Anokhin; Editorial board of journal «Izvestia visshikh uchebnikh zavedeniy. Yadernaya 